\title{
A Note on Equations for Steady-State Optimal Landscapes
}

\author{
HUI-HAI LIU \\ Earth Sciences Division \\ Lawrence Berkeley National Laboratory \\ Berkeley, CA 94720, USA \\ hhliu@lbl.gov
}

July 20, 2010 


\section{DISCLAIMER}

This document was prepared as an account of work sponsored by the United States Government. While this document is believed to contain correct information, neither the United States Government nor any agency thereof, nor The Regents of the University of California, nor any of their employees, makes any warranty, express or implied, or assumes any legal responsibility for the accuracy, completeness, or usefulness of any information, apparatus, product, or process disclosed, or represents that its use would not infringe privately owned rights. Reference herein to any specific commercial product, process, or service by its trade name, trademark, manufacturer, or otherwise, does not necessarily constitute or imply its endorsement, recommendation, or favoring by the United States Government or any agency thereof, or The Regents of the University of California. The views and opinions of authors expressed herein do not necessarily state or reflect those of the United States Government or any agency thereof or The Regents of the University of California.

Ernest Orlando Lawrence Berkeley National Laboratory is an equal opportunity employer. 


\begin{abstract}
Based on the optimality principle (that the global energy expenditure rate is at its minimum for a given landscape under steady state conditions) and calculus of variations, we have derived a group of partial differential equations for describing steady-state optimal landscapes without explicitly distinguishing between hillslopes and channel networks. Other than building on the well-established Mining's equation, this work does not rely on any empirical relationships (such as those relating hydraulic parameters to local slopes). Using additional constraints, we also theoretically demonstrate that steadystate water depth is a power function of local slope, which is consistent with field data.
\end{abstract}

Key words: Optimality; landscape modeling; USA 


\section{INTRODUCTION}

Mathematical modeling of landscapes (for drainage basins) has been an active research area in the hydrology community. A landscape consists of the two interrelated systems: the channel network and hillslopes (Rodriguez-Iturbe et al., 1992). Hillslopes generate water runoff resulting from rainfalls and the network collects water runoff through the channel reaches and transports it downstream. There exist approximately three classes of approaches for modeling (and/or characterizing) the morphology of a drainage basin associated with uniform lithology and minor structural control (Sun et al., 1995). The models in the first class are based on Shreve's fundamental stochastic postulate of random topology (Shreve, 1966, 1967). The second class is based on optimality principles (Howard, 1990; Rodriguez-Iturbe et al., 1992; Sun et al., 1995; Rinaldo et al., 2006). The third class is derived from a consideration of physical processes including erosion and deposition (e.g., Willgoose et al., 1991; Howard, 1994). The models based on optimality principles are particularly of interest, because similar principles seem to be able to explain a great number of complex natural phenomena that are determined by distinctly different processes (Bejan, 2000).

The role of optimality principles in forming complex natural patterns has been recognized for many years. Leopold and Langbein (1962) proposed a maximum entropy principle for studying the formation of landscapes. Howard (1990) developed a mathematical model (for optimal drainage networks) in which an initial network was generated by a random headward growth model, and then channels were shifted to minimize total stream power (or energy expenditure) within the network. The resulting networks are visually and morphometrically more similar to natural networks than the initial networks that are not subject to the minimized energy expenditure.

Rodriguez-Iturbe et al (1992) postulated principles of optimality in energy expenditure at both local and global scales for channel networks. The local optimality hypothesis states that networks will adjust their channel properties toward an optimal state in which the energy dissipation rate per unit channel area is constant throughout the network. The global optimality states that networks will adjust their topological structure such that the total energy dissipation rate is at the minimum within a given network. Based on these principles, Rinaldo et al. (1992) developed modeling approaches to generate optimal 
channel networks (OCNs) and compared their results with those from natural river basins. Striking similarity was observed for natural and optimal networks in their fractal aggregation structures and other relevant features. Positive comparisons between a variety of observations from natural channel networks and those derived from the optimality principles were also reported by Rodriguez-Iturbe et al (1992) and Molnar and Ramirez (1998), among others.

In this short communication, we propose a new theoretical framework for modeling optimal landscapes. While previous studies mainly use spatially "discrete” approaches as a result of considering energy dissipation through channel networks only, we develop a group of (partial differential) governing equations for steady-state optimal landscapes (including both channel networks and associated hillslopes) using calculus of variations (Weinstock, 1974). This paper is organized as follows. The next section will present the detailed derivations for these equations. A simplified case is then discussed to reveal that often observed power-function relationships between water depth and local slope could be theoretically obtained based on the optimality principles. Finally, the potential limitations and further improvements of our work will be discussed.

\section{THEORY}

We consider a landscape involving steady-state water flow and surface evolution processes. This assumption has been implicitly employed in previous studies on topological structures of channel networks (Howard, 1990; Rodriguez-Iturbe et al., 1992; Rinaldo et al., 2006). A land surface constantly responds to spatially and temporally variable forcing (such as rainfall). However, it develops average conditions (such as average hydraulic geometry) that are relatively stable on a large time scale (Leopold and Maddock, 1953; Molnar and Ramirez, 1998). Rinaldo et al. (2006) further indicated that several statistical properties are found to be almost the same for many rivers, irrespective of their age, supporting the steady-state treatment. Along the same line, rainfall is assumed to be in steady state and uniform through the landscape under consideration. While the model to be discussed can be extended to cases in which land properties (such

as those related to soil and vegetation) are spatially heterogeneous, For simplicity we focus on land surfaces with homogeneous properties. Although infiltration processes 
occurs during a rainfall event, they generally correspond to a relatively small portion of the rainfall water, especially during the period of heavy rainfalls that are expected to have important effects on landscape evolution processes. Therefore, infiltration is ignored in this study.

Based on the above simplifications, coupled water-flow (over a land surface) and surface-elevation equations can be derived from the principle that global energy expenditure rate is at the minimum. From the water mass (volume) conservation, steadystate water flow equation is given by

$$
\frac{\partial q_{x}}{\partial x}+\frac{\partial q_{y}}{\partial y}=Q
$$

where $x$ and $y$ are two horizontal coordinate axes, $q_{x}$ and $q_{y}\left(\mathrm{~m}^{2} / \mathrm{s}\right)$ are water fluxes (water velocity multiplied by water depth) along $x$ and $y$ directions, respectively, and $Q(\mathrm{~m} / \mathrm{s})$ is the rainfall rate.

Accordingly, energy expenditure rate for unit land-surface area, $\Delta E$, can be expressed as

$$
\Delta E=\frac{\partial\left(q_{x} E\right)}{\partial x}+\frac{\partial\left(q_{y} E\right)}{\partial y}-Q E
$$

The above equation simply states that for a given unit area, the energy expenditure rate at that location is equal to the energy carried by water flowing into the area minus the energy carried by water flowing out of the area. The rainfall is assumed to have the same energy as water at the location where the rain falls. The $E$ (a function of $x$ and $y$ ) represents the total energy including both potential (corresponding to elevation $z$ ) and kinetic energy:

$$
E=z+\frac{v^{2}}{2 g}
$$

where $g\left(\mathrm{~m}^{2} / \mathrm{s}\right)$ is gravitational acceleration. Note that the second term is generally small and has been ignored in some previous studies (e.g., Howard, 1990; Rinaldo et al., 2006). For completeness, this term is included here. 
A combination of Equations (1) and (2) yields

$\Delta E=q_{x} \frac{\partial E}{\partial x}+q_{y} \frac{\partial E}{\partial y}$

The water flux is generally given by Manning’s equation (Feng and Molz, 1997)

$$
\begin{aligned}
& q_{x}=-A S^{-1 / 4} \frac{\partial E}{\partial x} \\
& q_{y}=-A S_{*}^{-1 / 4} \frac{\partial E}{\partial y}
\end{aligned}
$$

where

$$
\begin{aligned}
& A=\frac{h^{5 / 3}}{n} \\
& S_{*}=S^{2}=\left(\frac{\partial E}{\partial x}\right)^{2}+\left(\frac{\partial E}{\partial y}\right)^{2}
\end{aligned}
$$

In Equation (5c), $h(\mathrm{~m})$ is water depth and $n$ is Manning coefficient. In Equation (5d), $S$ is called energy gradient that has been commonly approximated by elevation gradient (Feng and Molz, 1997). Note that Manning's equation was derived based on a consideration that water energy loss results from friction force only. In other words, the energy loss related to maintenance of channels (corresponding to sediment transport) (RodriguezIturbe et al., 1992) is not considered by Manning's equation. Although it is mathematically possible to consider such energy loss in determining water fluxes, previous studies seem to indicate that the current treatment is adequate for the purpose of flux calculations (Feng and Molz, 1997). One may also argue that during steady state and optimal conditions, energy loss related to sediment transport may be small. Nevertheless, this treatment is considered to be the first-order approximation only and further improvement may be possible in the future.

When we combine Equations (4) and (5), the global energy expenditure rate through domain $\Omega$ is given by

$$
\iint_{\Omega} \Delta E d x d y=\iint_{\Omega}\left(-A S_{*}^{3 / 4}\right) d x d y
$$


The optimality principle in our problem is to minimize the absolute value of the above integral. To do so, we employ calculus of variations that seeks optimal (stationary) solutions to a functional (a function of functions) by identifying unknown functions (Weinstock, 1974). For example, the former corresponds to the integral defined in Equation (6) and the latter to land-surface elevation distribution $z(x, y)$.

Based on Equations (1), (5) and (6), the Lagrange for the given problem is given by

$$
\begin{aligned}
& L=-A S_{*}^{3 / 4}+\lambda_{1}\left[-Q+\frac{\partial q_{x}}{\partial x}+\frac{\partial q_{y}}{\partial y}\right]+\lambda_{2}\left[S_{*}-\left(\frac{\partial E}{\partial x}\right)^{2}-\left(\frac{\partial E}{\partial y}\right)^{2}\right] \\
& +\lambda_{3}\left[q_{x}+A S^{-1 / 4} \frac{\partial E}{\partial x}\right]+\lambda_{4}\left[q_{y}+A S_{*}^{-1 / 4} \frac{\partial E}{\partial y}\right]
\end{aligned}
$$

Note that the first term is from Equation (6) and other terms are constraints from Equations (1) and (5). Use of these constraint terms allows considering related functions to be independent when determining the optimal solution to Equation (6). The $\lambda$ functions are Lagrange multipliers. A mathematically equivalent way to define $\mathrm{L}$ to avoid the use of some (or all) constrains is to directly insert Equations (1) and (5) into the first term of Equation (7). In this case, the number of independent functions will be reduced. However, the use of Equation (7) is more straightforward and easier to handle for the given problem.

The following Euler-Lagrange equation is used to determine an unknown function w associated with $L$ to minimize the integral defined in Equation (6) (Weinstock, 1974):

$$
\frac{\partial L}{\partial w}-\frac{\partial}{\partial x}\left(\frac{\partial L}{\partial w_{x}}\right)-\frac{\partial}{\partial y}\left(\frac{\partial L}{\partial w_{y}}\right)=0
$$

where $w_{x}$ and $w_{y}$ are partial derivatives with respect to $x$ and $y$ respectively. In this study, $w$ corresponds to $A, q_{x}, q_{y}, S_{*}$ and $E$, respectively. (Also note that application of the EulerLagrange equation to Lagrange multipliers will recover Equations (1) and (5).) 
Replacing $w$ with $A$ in Equation (8) and using the definition of $S_{*}$ (Equation (5d)), we obtain

$$
\left(\lambda_{3}-\frac{\partial E}{\partial x}\right) \frac{\partial E}{\partial x}+\left(\lambda_{4}-\frac{\partial E}{\partial y}\right) \frac{\partial E}{\partial y}=0
$$

Replacing $w$ with $q_{x}$ and $q_{y}$, respectively, in Equation (8) yields

$$
\begin{aligned}
& \lambda_{3}=\frac{\partial \lambda_{1}}{\partial x} \\
& \lambda_{4}=\frac{\partial \lambda_{1}}{\partial y}
\end{aligned}
$$

Replacing $w$ with $S *$ in Equation (8) and making use of Equation (9), we have

$$
\lambda_{2}=A S_{*}^{-1 / 4}
$$

Again, replacing $w$ with $E$ in Equation (8), letting $\lambda=\lambda_{1}-E$, and using Equation (1), we obtain

$$
\frac{\partial\left[A S_{*}{ }^{-1 / 4} \frac{\partial \lambda}{\partial x}\right]}{\partial x}+\frac{\partial\left[A S_{*}^{-1 / 4} \frac{\partial \lambda}{\partial y}\right]}{\partial y}=-Q
$$

By definition of $\lambda$, Equation (9) can be rewritten as

$\frac{\partial \lambda}{\partial x} \frac{\partial E}{\partial x}+\frac{\partial \lambda}{\partial y} \frac{\partial E}{\partial y}=0$

Combining Equations (1), (5a) and (5b) gives a new form of water flow equation:

$$
\frac{\partial\left[A S_{*}^{-1 / 4} \frac{\partial E}{\partial x}\right]}{\partial x}+\frac{\partial\left[A S_{*}^{-1 / 4} \frac{\partial E}{\partial y}\right]}{\partial y}=-Q
$$

A combination of Equations (12) to (14) corresponds to the minimization of the absolute value of global energy expenditure rate in Equation (6). The above three 
equations involve spatial distributions of three variables $(A, E$, and $\lambda)$, and therefore these distributions can be uniquely solved using these equations under appropriate boundary conditions. The exact physical meaning of the intermediate variable $\lambda$ remains to be found. As indicated in Equation (13), gradients of $E$ and $\lambda$ are perpendicular to each other. Equations (12) and (14) are also similar in form, although $S_{*}$ is directly related to $E$, rather than $\lambda$. Implications of these interesting features to topological structures of landscape need to be explored in the future. Elevation distributions $(z(x, y))$ can be obtained from $E(x, y)$ and water flow conditions from Equation (3). Note that the focus of this note is on the derivation of the equations for steady-state optimal landscapes (Equations (12) to (14)) whose validity can be justified by the mathematical rigor of the derivation procedure and the validity of the optimality principle. We will leave to future studies the development of procedures to numerically solve these equations.

It is of interest to compare the current study with those relating modeling landscape processes to calculus of variations (Sinclair and Ball, 1996; Peckham, 2003]. The chief difference between this work and those studies includes two aspects. First, the current study starts with the minimization of rigorously defined glob energy expenditure rate, whereas Sinclair and Ball (1996) and Peckham (2003) started with a given partial differential equation for landscape evolution, obtained from considering erosion and/or water flow processes based on several assumptions, to derive the corresponding (Lagrange) functional. However, the links between their derived functional and the energy expenditure is weak, and therefore physical interpretation of their functional remains to be found. Although the exact physical meaning of the intermediate variable $\lambda$ is not clear yet here, we believe that this can be resolved in the future, because it has a clear geometrical interpretation that its gradient is perpendicular to the energy gradient (Equation (13)). It is likely that $\lambda$ is closely related to the underlying erosion processes that are not explicitly considered herein. Second, in addition to the optimality of energy expenditure, our theory does not rely on empirical relationships except for the wellestablished Mining's equation, whereas those previous studies are based on either empirical power functions of hydraulic parameters (Peckham, 2003) or an assumption that erosion rate is proportional to the product of power functions for related parameters (Sinclair and Ball, 1996). 


\section{A SIMPLIFIED CASE}

While Equations (12) to (14) provide a general description for steady-state optimal landscapes, we study in this section a simplified case that gives some interesting closedform results. This simplified case also partially serves as a case study to demonstrate the usefulness of the general framework employed in this study. We consider two additional constraints for the optimization problem. First, we assume $A$ to be a function of local slope $S$ only. Many studies indicate that on average a number of hydraulic parameters including water depth $h$ (that is directly related to $A$ in Equation (5c)) can be considered as functions of local slope (Leopold and Maddock, 1953). Note that in general, $A$ should be considered a function of location, as discussed in Section 2. Secondly, we employ the following constraint:

$$
\int_{\Omega} E d x d y=C
$$

where $C$ is a constant. Since $E$ is mainly composed of potential energy $z$, the above equation essentially states that the average elevation through the model domain (or total volume of the landscape under consideration) remains unchanged, which is consistent with the steady-state assumption made in this study. It should be emphasized that the optimality principle corresponds to minimization of global energy expenditure rate, not the total energy within the model domain. Under steady state conditions, the global energy expenditure is equal to difference between the latter and energy carried by water flowing out of the system.

Along the same line to derive Equation (7), the Lagrange for this simplified problem is given by

$$
L=-A S_{*}^{3 / 4}+\lambda_{1}{ }^{*}\left[S_{*}-\left(\frac{\partial E}{\partial x}\right)^{2}-\left(\frac{\partial E}{\partial y}\right)^{2}\right]+\lambda_{2}^{*}[E-C]
$$

where the Lagrange multipliers $\lambda_{1}{ }^{*}$ and $\lambda_{2}{ }^{*}$ are a function of location and a constant, respectively. The last term on the right hand side of Equation (16) corresponds to the constraint defined in Equation (15). Note that the constraint related to water flow, 
Equation (14), is not included in Equation (16), but will be handled later for mathematical convenience.

Applying the Euler-Lagrange Equation (8) to $S_{*}$ gives

$\lambda_{1}^{*}=\frac{d\left(A S_{*}^{3 / 4}\right)}{d S_{*}}$

Applying the Euler-Lagrange Equation (8) to $E$ yields

$\lambda_{2}^{*}+\frac{\partial}{\partial x}\left(2 \lambda_{1}^{*} \frac{\partial E}{\partial x}\right)+\frac{\partial}{\partial y}\left(2 \lambda_{1}^{*} \frac{\partial E}{\partial y}\right)=0$

For the optimization results to be physically valid, they must satisfy the water flow equation (14). A direct comparison between Equations (14) and (18) reveals that they are identical under the following conditions

$\lambda_{1}^{*}=\left(\frac{\lambda_{2}^{*}}{2 Q}\right) A S_{*}^{-1 / 4}$

Combining Equations (17) and (19), we can obtain

$A \propto S_{*}^{-\frac{3}{4}+\frac{\lambda_{2}^{*}}{2 Q}}=S^{-\frac{3}{2}+\frac{\lambda_{2}^{*}}{Q}}$

From Equation (5c), water depth $h$ can be related to the local slope $S$ by $h \propto S^{-0.9+k}$

where $k$ is a constant related to rainfall and other conditions, and likely to be site specific.

Equation (21) shows that under the two additional constraints mentioned above, minimization of global energy expenditure rate results in a simple outcome, that water depth throughout the landscape is a power function of local slope. This theoretical result agrees very well with field observations for channel networks (e.g., Leopold and Maddock 1953). Leopold and Langbein (1962) indicate that on average, rivers in midwestern United States follow the relationship

$h \propto S^{-0.8}$ 
This corresponds $k=0.1$ in our Equation (21).

While some interesting results are obtained, it is important to keep in mind the limitations of the additional constraints used in this section. For example, $A$ (or water depth) depends not only on local slope, but also locations. This may explain why relations between observed recharge (related to water depth) and local slopes show relatively large degrees of fluctuations in many cases (e.g., Ijjasz-Vasquez and Bras, 1995). Nevertheless, the current treatment (that hydraulic parameters depend on local slopes only) has often been regarded as a good approximation in practice (Leopold and Maddock, 1953; Leopold and Langbein, 1962). Therefore, our results are relevant to practical applications. Also note that a combination of Equations (14) and (20) will result in a single partial differential equation describing steady-state landscape, which is essentially similar to that developed by Peckham (2003), though arrived at by a quite different line of reasoning.

\section{CONCLUDING REMARKS}

Based on the optimality principle and calculus of variations, this note derives a group of partial differential equations for describing steady-state optimal landscapes. They are developed for describing the whole landscapes without explicitly distinguishing between hillslopes and channel networks. Other than building on the well-established Mining's equation, the study does not rely on any empirical relationships (such as those relating hydraulic parameters to local slopes). Using additional constraints, we also demonstrate that water depth is a power function of local slope, which is consistent with field data.

However, this needs to be extended in future studies. First, the physical meaning of $\lambda$ in Equations (12) and (13) remains to be identified, while geometrically its gradient is perpendicular to the local energy gradient. Second, because the current study does not explicitly consider the erosion process (expected to be implicitly included in the optimality principle), the mathematical correlation between our equations and the corresponding erosion process is not totally clear at this point. Finally, numerical procedures need to be developed to solve the coupled equations - Equations (12) to (14). 


\section{REFERENCES}

Bejan, A. (2000) Shape and structure, from engineering to nature. Cambridge University Press.

Feng, K. \& Molz F.J. (1997) A 2-D diffusion-based wetland model. J. of Hydrology, 196, 230-250.

Howard, A.D. (1990) Theoretical model of optimal drainage networks. Water Resour. Res. 26(9), 2107-2117.

Howard, A.D. (1994) A detachment-limited model of drainage basin evolution. Water Resour. Res. 30(7), 2261-2285.

Leopold, L.B. \& Langbein, W.B. (1962) The concept of entropy in landscape evolution. U.S. Geol. Surv. Prof. Pap. 500-A.

Leopold, L.B. \& Maddock, T. (1953) The hydraulic geometry of stream channels and some physiographic implications. U.S. Geol. Surv. Prof. Pap. 252.

Molnar, P. \& Ramirez A. (1998) An analysis of energy expenditure in Goodwin Creek. Water Resour. Res. 34(7), 1819-1829.

Peckham, S.D. (2003) Mathmatical modeling of landforms: optimality and steady-state solutions. In: Conceptual and Modeling in Geomorphology: International Perspective (ed. By I.S. Evans, R. Dikau, E. Tokunaga, H. Ohmori \& M. Hirano), 167-182. TERRAPUB, Tokyo, Japan.

Rinaldo, A., Rodriguez-Iturbe, I., Rigon R., Bras, R.L., Ijjasz-vazquez, E. \& Marani A.(1992) Minimum energy and fractal structures of drainage networks. Water Resour. Res. 28, 2183-2191.

Rinaldo, A., Banavar, J. R. \& Maritan A. (2006) Trees, networks and hydrology. Water Resour. Res. 42, W06D07, doi:10.1029/2005WR004108.

Rodriguez-Iturbe, I., Rinaldo A., Rigon, A., Bras, R.L., Marani, A. \& Hijas-Vasquez, E. (1992) Energy dissipation, runoff production and the three-dimensional structure of river basins. Water Resour. Res. 28(4), 1095-1103.

Shreve, R. L. (1966) Statistical law of stream numbers. J. Geol., 75, 17-37.

Shreve, R. L. (1967) Infinite topologically random channel networks. J. Geol., 75, 397414.

Sinclair, K. \& Ball R.C. (1996) Mechanism for global optimization of river networks from local erosion rules. Phys. Rev. Lett. 76, 3360-3363. 
Sun, T., Meakin, P. \& Jossong T. (1995) Minimum energy dissipation river network with fractal boundaries. Physical Review E. 51(6), 5353-5359.

Weinstock R. (1974) Calculus of variations. Dover Publication, Inc., New York.

Willgoose, G.R., Bras, P.L. \& Rodriguez-Iturbe, I (1991) A coupled channel network growth and hillslope evolution model: 1. Theory. Water Resour. Res. 27, 1671-1684.

\section{Acknowledgement}

We are indebted to Drs. Yingqi Zhang and Dan Hawkes at Lawrence Berkeley National Laboratory for their critical and careful review of a preliminary version of this manuscript. This work was supported by the U.S. Department of Energy (DOE), under DOE Contract No. DE-AC02-05CH11231. 